\title{
Individuelle Herausforderung Ekzem
}

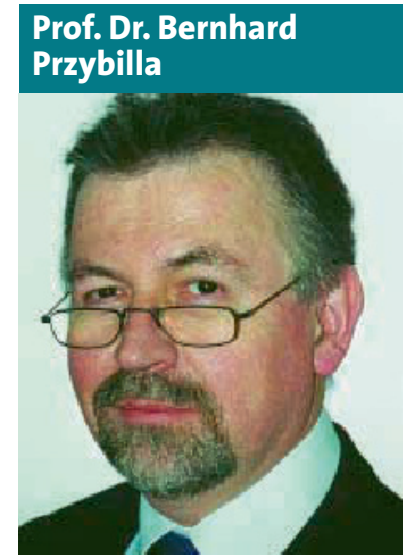

Klinik und Poliklinik für Dermatologie und Allergologie, Ludwig-MaximiliansUniversität, München, Sprecher der Sektion Dermatologie der DGAI

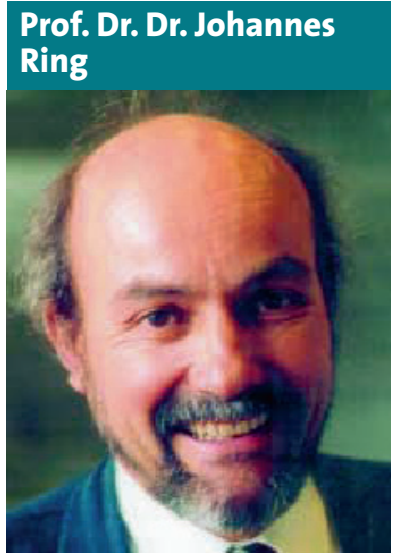

Klinik und Poliklinik für Dermatologie und Allergologie am Biederstein, Technische Universität München, Vizepräsident der DGAI solche Vielfalt nicht, nur atopisches Ekzem, Kontaktekzem - irritativ oder allergisch ausgelöst -, seborrhoisches Ekzem und nummuläres Ekzem sind zu unterscheiden.

Wegen ihrer hohen Prävalenz besonders bedeutsam sind atopisches Ekzem und Kontaktekzem. Nicht selten verlaufen sie chronisch, die Leiden des Patienten sind dann kaum zu überschätzen: Beschwerden durch die erkrankte Haut, psychische Beeinträchtigung, Einschränkungen in den sozialen Beziehungen, Behinderung am Arbeitsplatz - vor allem bei Befall der Hände -, finan-
„Atopisches Ekzem und Kontaktekzem können so chronisch oder rezidivierend verlaufen, dass sie als ,unheilbar' erscheinen mögen."
Abheilung - ein Beleg dafür, dass diese grundsätzlich erreicht werden kann! Nach Entlassung folgt dann allerdings oft rasch das Rezidiv ... Muss das so sein?

Bei Berücksichtigung von Hautphysiologie und Entzündungsmechanismen sowie nach Ermittlung und Ausschaltung individueller Provokationsfaktoren wird eine erfolgreiche Ekzemtherapie möglich! Besonders zu beachten ist, dass bei chronischem Ekzemverlauf meist sowohl Hautirritationen als auch allergische Reaktionen eine zielle Belastungen, zeitlicher Aufwand für die Therapie. Atopisches Ekzem und Kontaktekzem können so chronisch oder rezidivierend verlaufen, dass sie als „unheilbar“ erscheinen mögen. Zahlreiche Ärzte werden aufgesucht, aber auch die Anwendung immer wieder "neuer" Cremes oder Salben ist nicht oder nicht dauerhaft erfolgreich - oft werden „alternative" Therapieformen in Anspruch genommen. Klinikaufnahme mit konsequenter Therapie führt allerdings fast immer innerhalb von ein bis zwei Wochen zu weitreichender, ja vollständiger

Rolle spielen - nicht selten wird die Bedeutung der ersteren bei Kontaktekzem, der letzteren bei atopischem Ekzem unterschätzt! Durch individuell

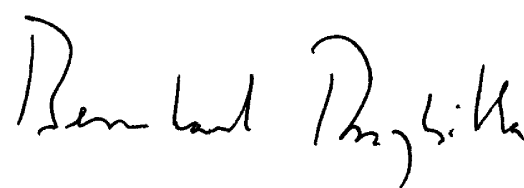

Prof. Dr. Bernhard Przybilla

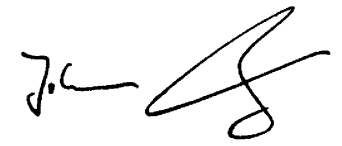

Prof. Dr. Dr. Johannes Ring angepasste Dermatotherapie, kenntnisreiche Allergiediagnostik und die Empfehlung praktischer Vorgehensweisen zur Meidung von erkannten Triggerfaktoren sind auch „schwierige“ Ekzeme „in den Griff zu kriegen“. Dazu sind aber unbedingt Verständnis und Mitarbeit des Patienten erforderlich, die nur durch aufwendige ärztliche $\mathrm{Zu}$ wendung erreicht und aufrechterhalten werden können. Eine wichtige Hilfe sind dabei formalisierte Schulungsprogramme, die seit einigen Jahren aufgebaut werden. Die ärztliche Betreuung des Patienten mit schwerer Ekzemerkrankung bleibt dabei aber immer eine individuelle Herausforderung. Die Kunst der Patientenführung muss sich hier bewähren!

Diese und die folgende Ausgabe von Allergo Journal wurden von der Sektion Dermatologie der Deutschen Gesellschaft für Allergologie und klinische Immunologie gestaltet und haben diese schwierige Krankheit zum Thema. Sie finden in Heft 7 Beiträge zum atopischen Ekzem, in Heft 8 zum Kontaktekzem. Die Themenhefte ersetzen kein Handbuch, sie versammeln ausgewählte Informationen $\mathrm{zu}$ wichtigen und aktuellen Aspekten von Ekzemerkrankungen - Kenntnisse zum Wohle unserer Patienten!

Mit den besten Grüßen 